\title{
Evolutionary Spectra Estimation of Field Measurement Typhoon Processes Using Wavelets
}

\author{
Guang-Dong Zhou, ${ }^{1}$ You-Liang Ding, ${ }^{2}$ and $\mathrm{Ai}-\mathrm{Qun} \mathrm{Li}^{2}$ \\ ${ }^{1}$ College of Civil and Transportation Engineering, Hohai University, Nanjing 210098, China \\ ${ }^{2}$ China Key Laboratory of Concrete and Prestressed Concrete Structures of Ministry of Education, \\ Southeast University, Nanjing 210096, China \\ Correspondence should be addressed to Guang-Dong Zhou; zhougd@hhu.edu.cn
}

Received 16 August 2014; Accepted 9 October 2014

Academic Editor: Ting-Hua Yi

Copyright (C) 2015 Guang-Dong Zhou et al. This is an open access article distributed under the Creative Commons Attribution License, which permits unrestricted use, distribution, and reproduction in any medium, provided the original work is properly cited.

\begin{abstract}
This paper presents a wavelet-based method for estimating evolutionary power spectral density (EPSD) of nonstationary stochastic oscillatory processes and its application to field measured typhoon processes. The EPSD, which is deduced in a closed form based on the definition of the EPSD and the algorithm of the continuous wavelet transform, can be formulated as a sum of squared moduli of the wavelet functions in time domain modulated by frequency-dependent coefficients that relate to the squared values of wavelet coefficients and two wavelet functions with different time shifts. A parametric study is conducted to examine the efficacy of the wavelet-based estimation method and the accuracy of different wavelets. The results indicate that all of the estimated EPSDs have acceptable accuracy in engineering application and the Morlet transform can provide desirable estimations in both time and frequency domains. Finally, the proposed method is adopted to investigate the time-frequency characteristics of the Typhoon Matsa measured in bridge site. The nonstationary energy distribution and stationary frequency component during the whole process are found. The work in this paper may promote an improved understanding of the nonstationary features of typhoon winds.
\end{abstract}

\section{Introduction}

Wind characteristics provide indispensable information for predicting structural dynamic responses and have been comprehensively investigated for many years through statistical methodology, signal processing technology, and field measurement. Conventional studies assume that the boundarylayer wind speed is a stationary ergodic random process consisting of a constant mean wind and three fluctuating wind components with the Gaussian distributions. However, the sudden and sporadic behaviors exhibit in many field measurement typhoon processes, especially during the phases of typhoon coming, typhoon leaving, and typhoon eye passing, because the air flow is thoroughly disturbed when it attacks the obstacles on the ground $[1,2]$. The assumption of an ergodic or stationary random process may be not applicable in many typhoon records. Therefore, it is more realistic to model typhoon winds as a nonstationary random process, whose energy distribution and frequency content change with time.

The nonstationarity of boundary layer winds has been explored by many researchers. Early in 1983, Lin and Yang have suggested that the nonstationary turbulence wind can be modeled by a stationary stochastic process multiplied with a determined envelope function [3]. However, because of the high computational cost of nonlinear analysis of structural wind-induced vibration, the theory of nonstationary wind processes develops slowly. With the development of signal processing technologies, the study involving nonstationary winds has been remotivated. Smith and Mehta have used autoregressive (AR) models to investigate nonstationary wind data collected in the field to overcome the limitation of the stationary wind model [4]. Gurley and Kareem have presented the application of a wavelet transform in winds and concluded that the wavelet analysis could characterize the transit nature of convective turbulence in hurricane wind [5]. 
The structural health monitoring (SHM) system, which could monitor actual wind speed in structural site, provides critical information for studying nonstationary winds [6, 7]. Xu et al. have modeled typhoon winds as a time-varying mean wind speed plus a well-behaved fluctuating wind speed admitted as a stationary random process with a Gaussian distribution [8, 9]. This concept was also applied in modeling the Hurricane Lili by Wang and Kareem [10], the typhoon by Li and Wu [11], and the gust-front winds by Kwon and Kareem [12]. Chen and Letchford have proposed a three-layer model for describing nonstationary winds resulting from downbursts, where the three layers represent time-varying mean wind speed, timevarying variance, and time-varying spectral content [13]. The fluctuating wind speed components of downbursts were characterized by a time-varying AR model [14] or wavelets [15]. In fact, the remaining fluctuating wind speed component of a typhoon process may still possess time-varying frequency characteristics after subtracting the time-varying mean wind speed. Therefore, treating the fluctuating wind speed as a zero-mean nonstationary oscillatory process is more reasonable $[13,16,17]$.

Being different from stationary random processes, the frequency content of the nonstationary fluctuating wind changes in time and cannot be accurately described by the power spectral density (PSD) that calculates the mean composition during the whole process. Many methods have been proposed to estimate the time-dependent spectra of nonstationary random processes, such as the Wigner-Ville method (WVM), the short-time Fourier transform (STFT), the S-transform (ST), and the wavelet transform. The WVM, STFT, and ST show some intrinsic drawbacks relating low resolution and energy leakage [18-22]. The wavelet transform, which has the capability of focusing on the multiscale details of a signal by a series of moveable and scalable time-frequency windows, provides a promising tool to explore signal features in both the time and frequency domains [23-27]. Spanos and Failla have introduced an innovated wavelets-based method to estimate the evolutionary power spectral density (EPSD) of seismic motions [28]. In this work, the rigorous derivation has been conducted and the EPSD was represented as a sum of time-independent shape functions, which is equal to the squared moduli of the Fourier transforms of the wavelets associated with the selected scales, modulated by time-dependent coefficients. Subsequently, the method has been expanded to arbitrary nonstationary processes by Failla et al. [20]. This method has been also applied in nonstationary downburst winds by Huang and Chen [16]. The results indicate that the wavelets-based method can capture the time-frequency characteristics of the downburst wind fluctuations.

In this paper, a wavelet-based method is firstly deduced in a closed form to estimate the EPSD of nonstationary typhoon processes. In the method, the EPSD is constructed by closed-form representations of wavelets in time domain, which can break the restriction in the method developed by Spanos and Failla that the analytical frequency-dependent wavelet functions are required. Then, a parametric study is implemented to examine the efficacy of the proposed approach and the influence of wavelet functions on the accuracy of the estimations. At last, the proposed method is applied to estimate the EPSD of field measured typhoon winds to promote an improved understanding of the timefrequency characteristics of nonstationary typhoon winds.

\section{EPSD Estimation Using Wavelet Transform}

The EPSD, which was introduced by Priestley in 1967, is a time-dependent analogue of the spectrum of a stationary stochastic process and valid for a class of zero-mean nonstationary oscillatory processes. The EPSD describes the local power-frequency distribution at each instant of time; therefore, it preserves the physical interpretation of the spectrum of a stationary stochastic process as a powerfrequency distribution $[29,30]$.

Priestley has suggested that the nonstationary stochastic process $y(t)$ can be defined by the Riemann-Stieltjes (R-S) integration [30]

$$
y(t)=\int_{-\infty}^{+\infty} A(\omega, t) e^{i \omega t} d Z(\omega),
$$

where $A(\omega, t)$ denotes the modulating function, $i$ represents the imaginary unit, and $Z(\omega)$ denotes a complex random process with orthogonal increments. Then, the EPSD of the nonstationary stochastic process $y(t)$ can be calculated by

$$
S_{y y}(\omega, t)=|A(\omega, t)|^{2} S_{x x}(\omega),
$$

where $S_{y y}(\omega, t)$ is the EPSD of nonstationary stochastic process $y(t)$ and $S_{g g}(\omega)$ represents the time-independent power spectral density of $x(t)$. The EPSD has two typical marginal features as follows:

$$
\begin{gathered}
\int_{-\infty}^{+\infty} S_{y y}(\omega, t) d t=\left|\bar{S}_{y y}(\omega)\right|^{2}, \\
\int_{0}^{+\infty} S_{y y}(\omega, t) d \omega=|y(t)|^{2},
\end{gathered}
$$

where $\bar{S}_{y y}(\omega)$ represents the time-independent power spectral density of $y(t)$.

The continuous wavelet transform (CWT) of a nonstationary stochastic process $y(t)$ in frequency domain is defined as

$$
W_{y}(a, b)=\frac{\sqrt{a}}{2 \pi} \int_{-\infty}^{\infty} \Psi^{*}(a \omega) e^{i \omega b} Y(\omega) d \omega,
$$

where $W_{y}(a, b)$ represents the wavelet coefficients, $a$ and $b$ denote the scale parameter and shift parameter, respectively, $Y(\omega)$ is the Fourier transform of $y(t), \Psi(\omega)$ means the wavelet function in frequency domain, and the symbol $(*)$ is the complex conjugate. $a$ and $b$ are discretized by $a_{r}=a_{0}{ }^{r}(r=$ $1,2, \ldots, m)$ and $b_{j}=(j-1) b_{0}(j=1,2, \ldots, n)$, respectively, in which $a_{0}$ and $b_{0}$ are constants, $m$ is the total number of discrete scales, and $n$ is the total number of time intervals retained in the discrete process $y(t)$. For a specified time instant $b_{j}$, (5) can be rewritten as

$$
W_{y}\left(a, b_{j}\right)=\frac{\sqrt{a}}{2 \pi} \int_{-\infty}^{\infty} \Psi^{*}(a \omega) e^{i \omega b_{j}} \int_{-\infty}^{\infty} y(t) e^{-i \omega t} d t d \omega .
$$


Since the time-varying rate of the modulation function of practical signals in civil engineering is slow as compared to the localized wavelet functions, it can be assumed that the scale $a$ has one-to-one corresponding relation with frequency $\omega$ in the wavelet transform. Then, the wavelet coefficients are equal to

$$
\begin{aligned}
& W_{y}\left(a, b_{j}\right) \\
& \approx \frac{\sqrt{a}}{2 \pi} \int_{-\infty}^{\infty} \Psi^{*}(a \omega) e^{i \omega b_{j}} \iint_{-\infty}^{\infty} A(a, t) e^{i a t} d Z(a) e^{-i \omega t} d t d \omega \\
& =\iint_{-\infty}^{\infty} A(a, t) \frac{\sqrt{a}}{2 \pi} \\
& \quad \times \int_{-\infty}^{\infty} \Psi^{*}(a \omega) e^{-i \omega t} e^{i \omega b_{j}} d \omega e^{i a t} d Z(a) e^{i a t} e^{-i a t} d t \\
& =\int_{-\infty}^{\infty} f(t) e^{-i a t} d t,
\end{aligned}
$$

where

$$
\begin{aligned}
f(t) & =\int_{-\infty}^{\infty}\left[A(a, t) \frac{1}{\sqrt{a}} \psi\left(-\frac{t-b_{j}}{a}\right)\right] e^{i a t} e^{i a t} d Z(a) \\
& =\int_{-\infty}^{\infty} H(a, t) e^{i a t} d Z(a) .
\end{aligned}
$$

In (8), $f(t)$ is a new nonstationary stochastic process defined by the nonuniform modulating function $H(a, t)$. The $H(a, t)$ is equal to the wavelet function in time domain at time instant $b_{j}$ multiplied by the nonuniform modulating function $A(a, t)$ of the original nonstationary process $y(t)$. Therefore, the wavelet coefficient at a given instant $b_{j}, W_{y}\left(a, b_{j}\right)$, can be interpreted as the Fourier spectrum of the nonstationary stochastic process $f(t)$.

By using the marginal property of the EPSD, the following equation can be obtained

$$
\begin{aligned}
\left|W_{y}\left(a, b_{j}\right)\right|^{2} & =\int_{-\infty}^{\infty} S_{f f}(a, t) d t \\
& =\int_{-\infty}^{\infty}|A(a, t)|^{2}\left|\frac{1}{\sqrt{a}} \psi\left(-\frac{t-b_{j}}{a}\right)\right|^{2} S_{x x}(a) d t \\
& =\int_{-\infty}^{\infty}\left|\frac{1}{\sqrt{a}} \psi\left(-\frac{t-b_{j}}{a}\right)\right|^{2} S_{y y}(a, t) d t
\end{aligned}
$$

As a result, a closed-form expression between the instantaneous mean squared values of wavelet coefficients at a given time instant and the EPSD of the process $y(t)$ is deduced. For all time instants, (9) can be deployed as

$$
\begin{aligned}
E\left[\left|W_{y}\left(a, b_{1}\right)\right|^{2}\right] & =\int_{-\infty}^{\infty}\left|\frac{1}{\sqrt{a}} \psi\left(-\frac{t-b_{1}}{a}\right)\right|^{2} S_{y y}(a, t) d t, \\
E\left[\left|W_{y}\left(a, b_{2}\right)\right|^{2}\right] & =\int_{-\infty}^{\infty}\left|\frac{1}{\sqrt{a}} \psi\left(-\frac{t-b_{2}}{a}\right)\right|^{2} S_{y y}(a, t) d t, \\
& \vdots \\
E\left[\left|W_{y}\left(a, b_{n}\right)\right|^{2}\right] & =\int_{-\infty}^{\infty}\left|\frac{1}{\sqrt{a}} \psi\left(-\frac{t-b_{n}}{a}\right)\right|^{2} S_{y y}(a, t) d t .
\end{aligned}
$$

The general solution to (10) is

$$
S_{y y}(a, t)=\sum_{k=1}^{n} \gamma_{j}(a)\left|\frac{1}{\sqrt{a}} \psi\left(-\frac{t-b_{k}}{a}\right)\right|^{2} \quad k=1,2, \ldots, n,
$$

where $\gamma_{j}(a)$ is frequency-dependent coefficient and determined by

$$
\left[\begin{array}{cccc}
\delta_{1,1} & \delta_{1,2} & \cdots & \delta_{1, n} \\
\delta_{2,1} & \delta_{2,2} & \cdots & \delta_{2, n} \\
\vdots & \vdots & \vdots & \vdots \\
\delta_{n, 1} & \delta_{n, 2} & \cdots & \delta_{n, n}
\end{array}\right]\left[\begin{array}{c}
\gamma_{1}(a) \\
\gamma_{2}(a) \\
\vdots \\
\gamma_{n}(a)
\end{array}\right]=\left[\begin{array}{c}
E\left[\left|W_{y}\left(a, b_{1}\right)\right|^{2}\right] \\
E\left[\left|W_{y}\left(a, b_{2}\right)\right|^{2}\right] \\
\vdots \\
E\left[\left|W_{y}\left(a, b_{n}\right)\right|^{2}\right]
\end{array}\right],
$$

where

$$
\begin{aligned}
\delta_{k, j} & =\int_{-\infty}^{\infty}\left|\frac{1}{\sqrt{a}} \psi\left(-\frac{t-b_{k}}{a}\right)\right|^{2}\left|\frac{1}{\sqrt{a}} \psi\left(-\frac{t-b_{j}}{a}\right)\right|^{2} d t \\
& =\int_{-\infty}^{\infty} U_{k, j}\left(a, b_{k}, b_{j}, t\right) d t .
\end{aligned}
$$

Therefore, the EPSD of fluctuating wind speed can be estimated using (11), (12), and (13), which provides a powerful alternation of the approach proposed by Spanos and Failla [28]. The EPSDs defined by the wavelets in frequency domain in [28] and the EPSD in this paper are estimated by a series of wavelets in time domain. These formulas rely on the property that the wavelet coefficients of a nonstationary process at a certain time instant can be treated as a stochastic process with EPSD given in terms of the EPSD of the process in a closed form. This yields that the target EPSD is equal to a sum of time-dependent shape functions modulated by frequency-dependent coefficients. The shape functions are the squared moduli of the wavelet functions in time domain during the whole duration, and the coefficients relate to the squared values of wavelet coefficients and two wavelet functions with different time shifts. The procedure of wavelet-based EPSD estimation proposed in this paper is shown in Figure 1. Firstly, choose appropriate wavelet function and implement wavelet transform to obtain wavelet coefficients with selected scales and different time instants; 


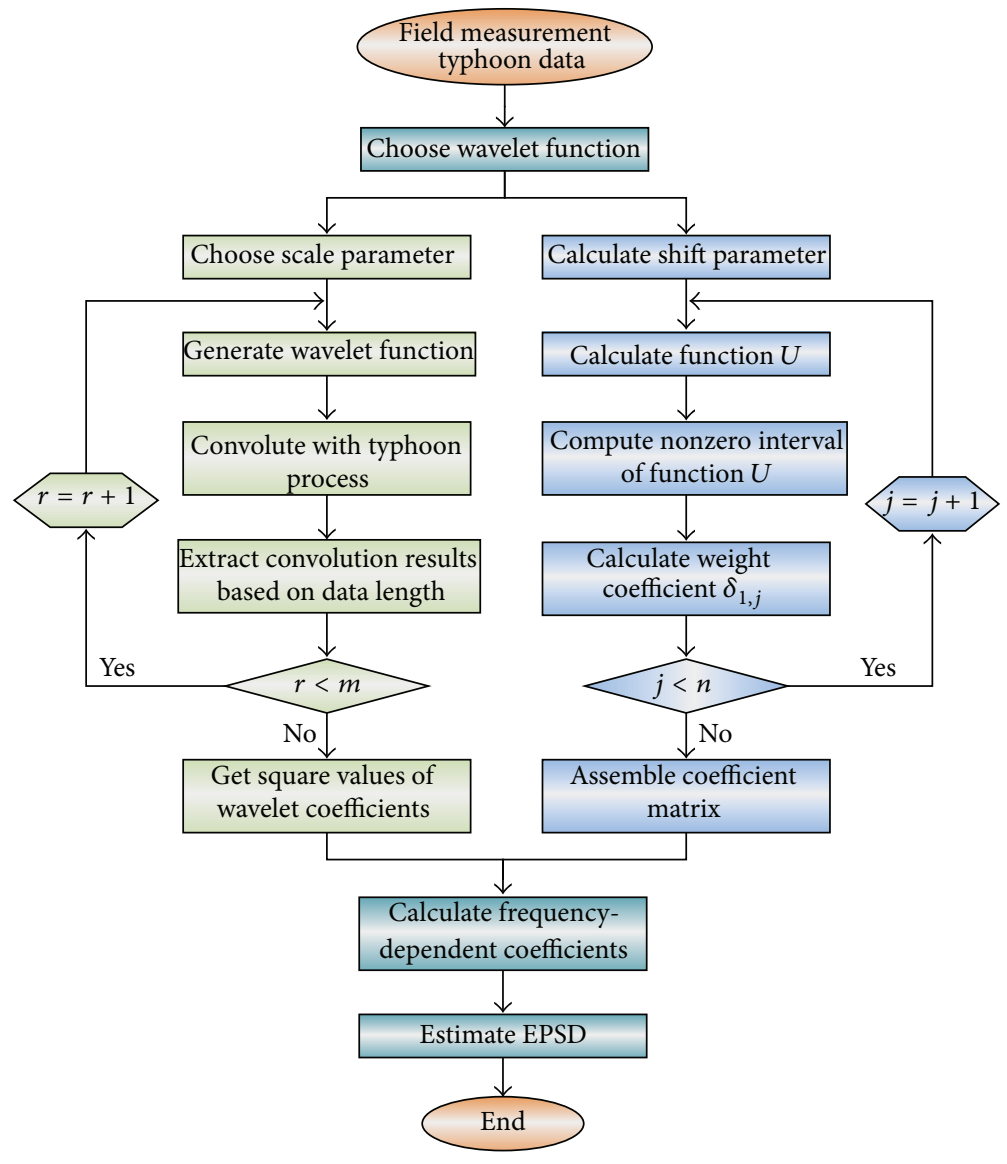

FIGURE 1: Flow chart of wavelet-based EPSD estimation.

then, calculate frequency-dependent coefficients by solving (12) for all scales; at last, estimate the EPSD of the process $y(t)$ by assembling the frequency-dependent coefficients and time-dependent wavelet functions. Because of the property of compact support, the nonzero portion of wavelet function in time domain is short. The element $\delta_{k, j}$ is equal to zero when the time interval, $\left(b_{k}-b_{j}\right)$, exceeds the support range of the wavelet function. Thus, the coefficient matrix in (13) is a zonal symmetric matrix. It should be pointed out that all theoretical formulations are derived based on the definition of nonstationary oscillatory processes. Therefore, the results in this paper are restricted in oscillatory processes. No detailed limitation about the wavelet basis is employed, so any wavelet whose function in time domain exists can be applied in the method proposed in this paper.

\section{Parametric Study}

Because the frequency localization properties and the time localization characteristics of different wavelet functions are discrepant, their capabilities of capturing time-frequency features of a signal are nonuniform. Thus, selecting a suitable wavelet function for the EPSD estimation of the nonstationary wind speed is an important issue. The predicting of structural wind-induced responses requires not only the amplitude but also the frequency content of fluctuating wind; therefore, the high resolution of the wavelet function in both time and frequency domains is critical. But according to the Heisenberg uncertainty principle, the time resolution and the frequency resolution are inconsistent. It is impossible that a wavelet function simultaneously has highest resolution in time domain and in frequency domain, so the compromise solution that selects a wavelet function with acceptable resolution is the only choice. In order to verify the effectiveness of the proposed method and assess the accuracy of different wavelet functions, three types of "mother" wavelets including the Morlet wavelet (MO), the Mexican Hat wavelet (MH), and the Littlewood-Paley wavelet (L-P) are employed herein.

The simulated nonstationary typhoon processes with determined EPSD are adopted. The simulated nonstationary typhoon processes are synthesized by stationary stochastic wind processes modulated by a modulating function [3]. The stationary stochastic wind processes are described by the Simiu spectra, and the modulating function consults the modulating function in earthquake engineering. As a result, the EPSD used for simulating nonstationary typhoon processes is

$$
S_{y y}(\theta, t)=\left|\exp \left(-\frac{(t-200)^{2}}{18000}\right)\right|^{2} \frac{200\left(u^{*}\right)^{2} Z / V}{(1+50 \theta Z / V)^{5 / 3}},
$$




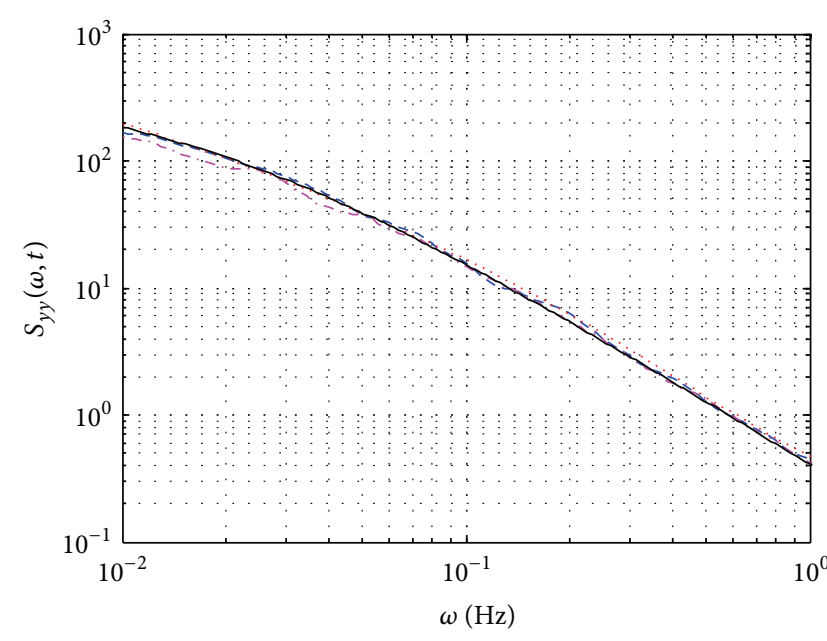

(a) $t=125 \mathrm{~s}$

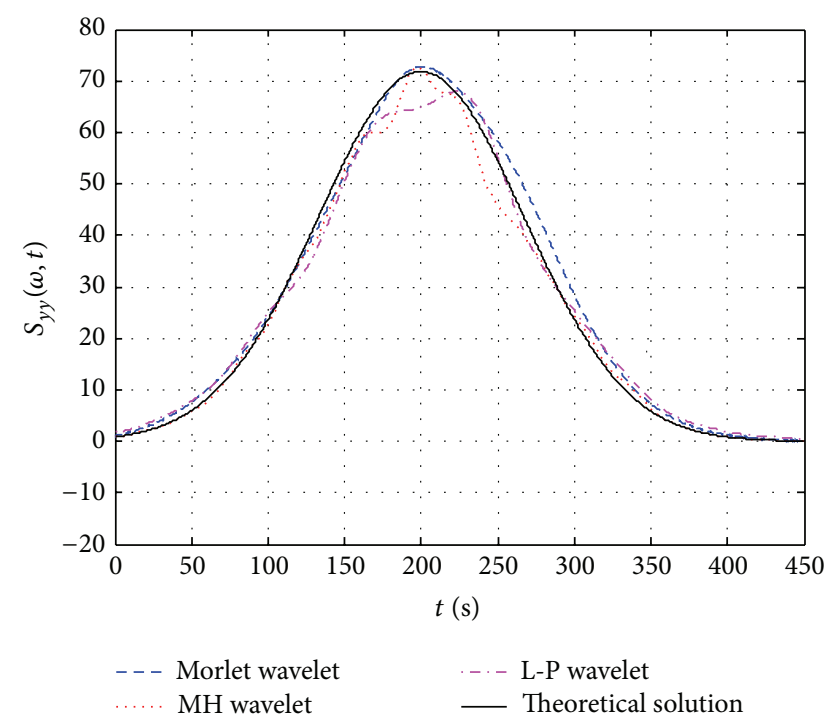

(c) $\omega=0.04 \mathrm{~Hz}$

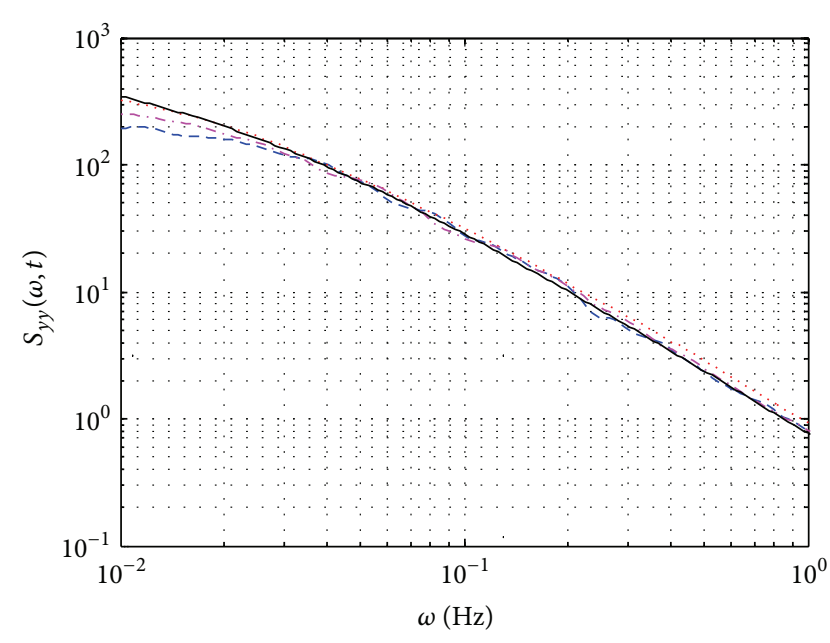

(b) $t=200 \mathrm{~s}$

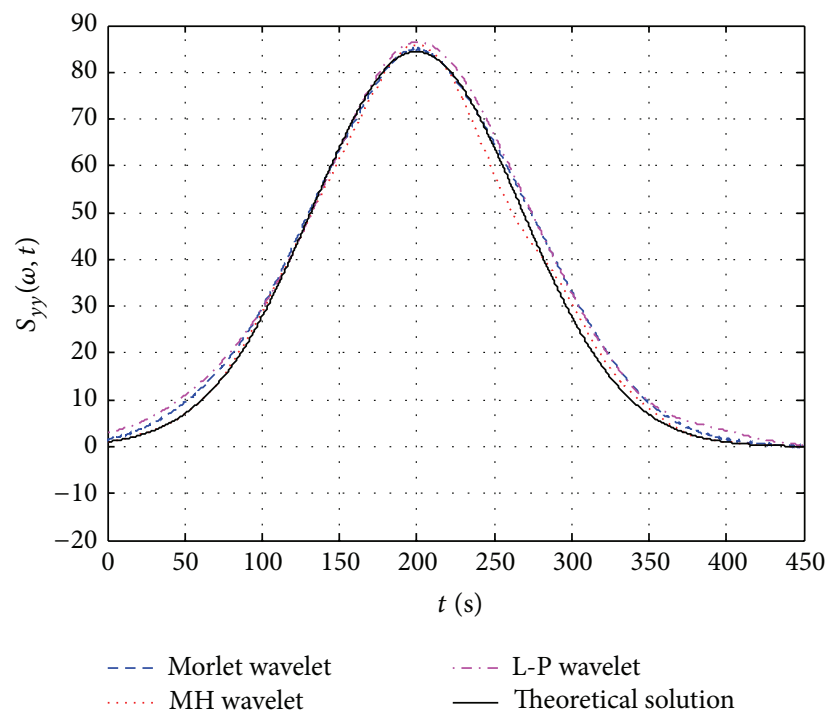

(d) $\omega=0.05 \mathrm{~Hz}$

FIGURE 2: Comparison of estimated EPSDs with theoretical solution.

where $\theta$ denotes the frequency with the unit of $\mathrm{Hz}, u^{*}$ represents the wind friction speed, $Z$ is the height of the wind speed relative to sea level, and $V$ implies the mean wind speed.

The $450 \mathrm{~s}$ and $4 \mathrm{~Hz}$ are set as the simulated wind duration and the sampling rate, respectively. The stationary stochastic wind processes are simulated by the classical spectral representation method [31, 32]. The synthesis is run 500 times, and the mean square values of the wavelet coefficients in (12) are calculated. The estimated EPSDs using the three wavelet functions are shown in Figure 2. To conduct comparison between the estimated EPSDs and the theoretical solution, the estimated EPSDs are sliced at different time instants and different frequency points, which are named as time slices and frequency slices in this paper, respectively. From those figures, it can be seen that the EPSDs estimated by the three wavelet functions show excellent agreement with the theoretical solution in both time and frequency domains, which demonstrates that the proposed method can estimate the EPSDs of nonstationary typhoon processes with acceptable reliability.

For further comparing the ability of the three wavelet functions in capturing the time-frequency characteristics, the estimating errors of the three wavelet functions are listed in Table 1. In the table, the error at a time instant is defined by the sum of the squared differences between the common logarithm of the estimated EPSD and the common logarithm of the theoretical solution, and the error at a frequency point is defined by the sum of the squared differences between the estimated EPSD and the theoretical solution. In time domain, the L-P transform shows the highest accuracy when compared with the other two wavelet functions, and, in frequency domain, the $\mathrm{MH}$ provides the most reliability. In frequency domain, the errors of the $\mathrm{MO}$ transform are bigger than those of the L-P transform and smaller than those of the $\mathrm{MH}$ transform. However, in time domain, the errors of the MO transform are smaller than those of the L-P transform 
TABLE 1: Estimating error using three wavelet functions.

\begin{tabular}{lcccccc}
\hline & \multicolumn{2}{c}{ Time slice } & & & \multicolumn{2}{c}{ Frequency slice } \\
Time & MO & MH & L-P & Frequency & MO & MH \\
\hline$t=125 \mathrm{~s}$ & 0.0033 & 0.0078 & 0.0086 & $\omega=0.04 \mathrm{~Hz}$ & 5.196 & 4.526 \\
$t=200 \mathrm{~s}$ & 0.0321 & 0.0447 & 0.0108 & $\omega=0.05 \mathrm{~Hz}$ & 5.730 & 4.943 \\
$t=250 \mathrm{~s}$ & 0.0129 & 0.0216 & 0.0109 & $\omega=0.1 \mathrm{~Hz}$ & 0.561 & 0.422 \\
$t=300 \mathrm{~s}$ & 0.0112 & 0.0136 & 0.0072 & $\omega=0.2 \mathrm{~Hz}$ & 0.167 & 0.129 \\
$t=400 \mathrm{~s}$ & 0.0126 & 0.0217 & 0.0114 & $\omega=0.5 \mathrm{~Hz}$ & 0.0057 & 0.0042 \\
\hline
\end{tabular}

and bigger than those of the $\mathrm{MH}$ transform. The underlying reasons are that the window of the $\mathrm{MH}$ in time domain is narrower that the other two wavelets and the window of the L-P in frequency domain is narrower that the other two wavelets. Combining the observation ability in both time and frequency domains, the MO transform is the most suitable choice among the three wavelet functions to conduct EPSD estimation of nonstationary typhoon processes.

\section{EPSD of Field Measurement Typhoon Process}

The Runyang Suspension Bridge (RSB), as shown in Figure 3, is a hinged and simply supported steel box-girder bridge with a main span of $1490 \mathrm{~m}$ and two side spans, each of $470 \mathrm{~m}$. The dynamic analysis finds that the bridge has extremely low stiffness and is susceptible to strong winds. The meteorological study indicates that the RSB is located in the northern region of the subtropical zone with typical subtropical monsoon climate characteristics. Many adverse weather events including typhoons, storms, and thunderstorms have happened in this region. Therefore, it is essential to conduct researches about the typhoon processes in bridge site and their effects on structural safety.

4.1. Typhoon Monitoring. Wind loads and wind-induced vibrations of large-scale structures with low stiffness are concerned by researchers all over the world [33, 34]. A sophisticated SHM system, which could report in realtime the structural condition and enable operational cost reduction by performing prognostic and preventative maintenance [35], has been permanently deployed on the RSB during construction. Two WA15 anemometers produced by the Vaisala Company (USA) are integrated in the SHM system. One anemometer is placed in the middle of the main span (upstream) and $69.300 \mathrm{~m}$ above ground, and the other anemometer locates on the top of the south tower (downstream) and $218.905 \mathrm{~m}$ above ground. The WA15 anemometer and its location are displayed in Figure 4. For the airflow is disturbed by the bridge deck easily when the wind attacks the bridge, the anemometer should have enough distance from the bridge to obtain the real wind characteristics data. The anemometer is fixed apart from the bridge about $9 \mathrm{~m}$. The two anemometers are installed to the north, with the angle of the wind direction defined by $0^{\circ}$ corresponding to the north. By the convention that positive rotation goes clockwise, the wind from east has an angle of $90^{\circ}$, and so on. The anemometer

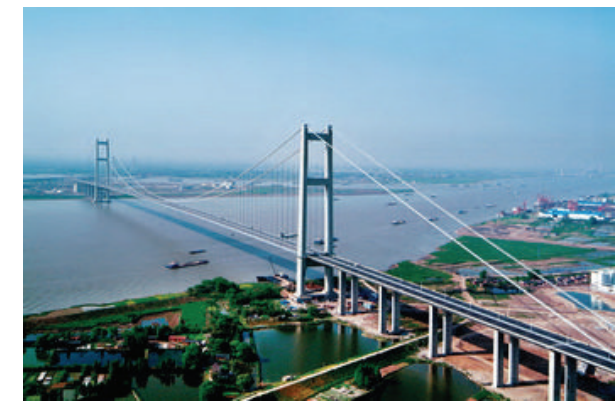

FIgURE 3: Overview of the Runyang Suspension Bridge.

can work in the temperature range of $-55-55^{\circ} \mathrm{C}$. The range of wind speed measurement is set from $0.4 \mathrm{~m} / \mathrm{s}$ to $51.2 \mathrm{~m} / \mathrm{s}$, and its resolution is less than $0.1 \mathrm{~m} / \mathrm{s}$. The sampling frequency is set as $1 \mathrm{~Hz}$. The recorded data includes real-time horizontal wind speed and wind direction. In August 2005, the Typhoon Matsa crossed over the RSB; the SHM system recorded the typhoon process both in the middle span and on the tower top. After subtracting the mean wind component, the time histories of fluctuating winds of the Typhoon Matsa are shown in Figure 5. In the two positions, the fluctuating wind speed is small and not more than $5 \mathrm{~m} / \mathrm{s}$ before and after the typhoon passing. However, the fluctuating wind speed increases dramatically during the Typhoon Matsa passing. The maximum fluctuating wind speed is close to $20 \mathrm{~m} / \mathrm{s}$ on tower top.

4.2. Estimated EPSD Validation. Using the wavelet-based method proposed in this paper, the EPSDs of the Typhoon Matsa measured in middle span and on tower top are estimated by the MO transform. Because the exact EPSDs of the two field measurement typhoon processes are unknown, it is difficult to directly validate the reliability of the estimated results. It is well known that the PSD of a signal can be calculated by many well-accepted algorithms, so the marginal feature that the integral of EPSD with time can be interpreted as the square of the time-independent power spectral density is adopted to verify the estimated results. According to (3), the integration of EPSD with time is equal to the square of PSD. Using this rule, the estimated EPSDs are integrated with time and compared with the PSD estimated by the Fourier transform, as displayed in Figure 6. It can be found that the integrations of EPSDs agree well with the PSDs in the two locations, which verifies the reliability of the estimated EPSDs. 


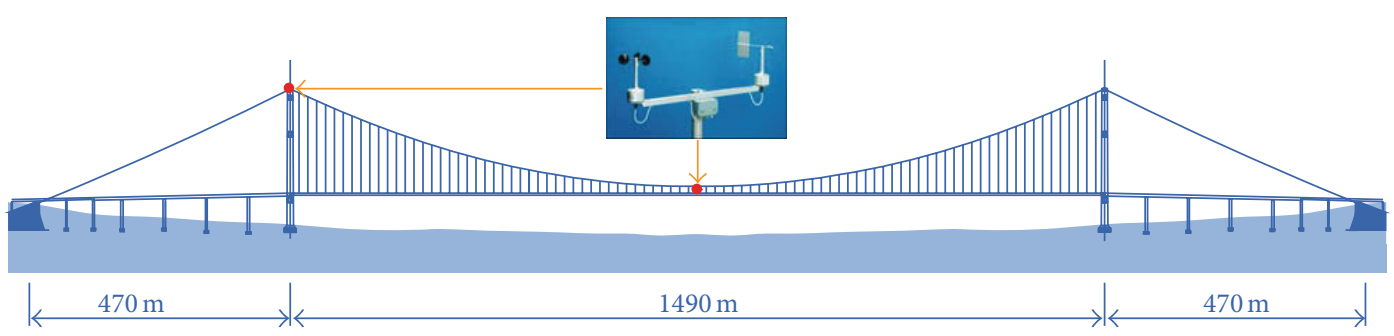

FIGURE 4: WA15 anemometer and its location.

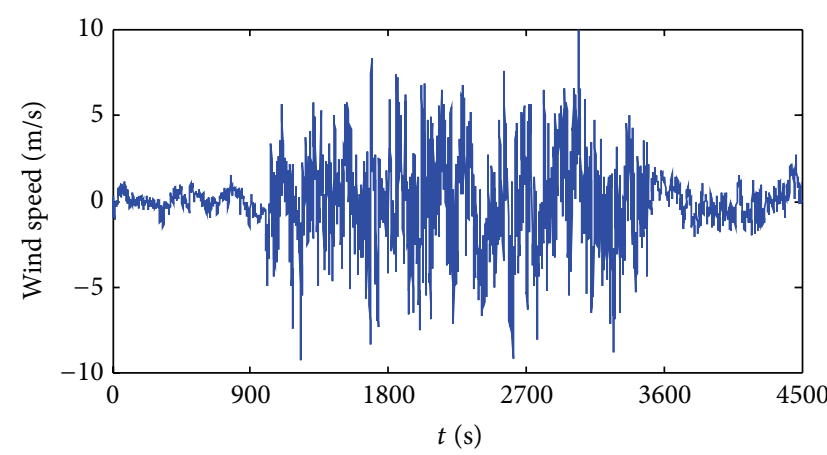

(a) Middle span

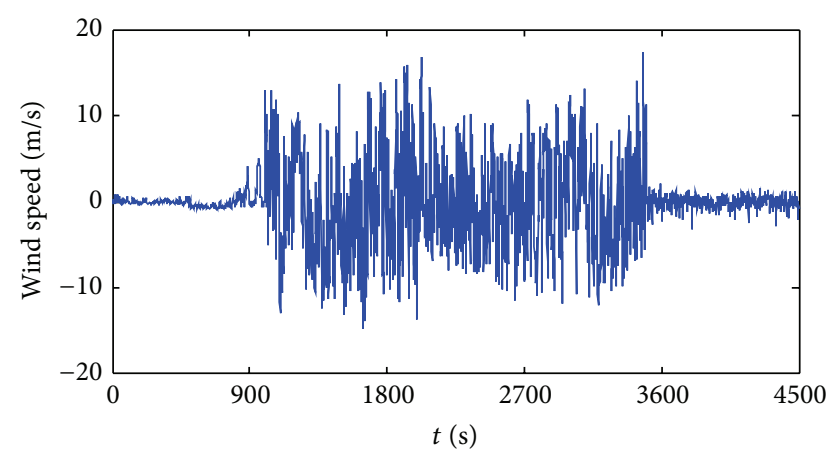

(b) Tower top

Figure 5: Time histories of the Typhoon Matsa.

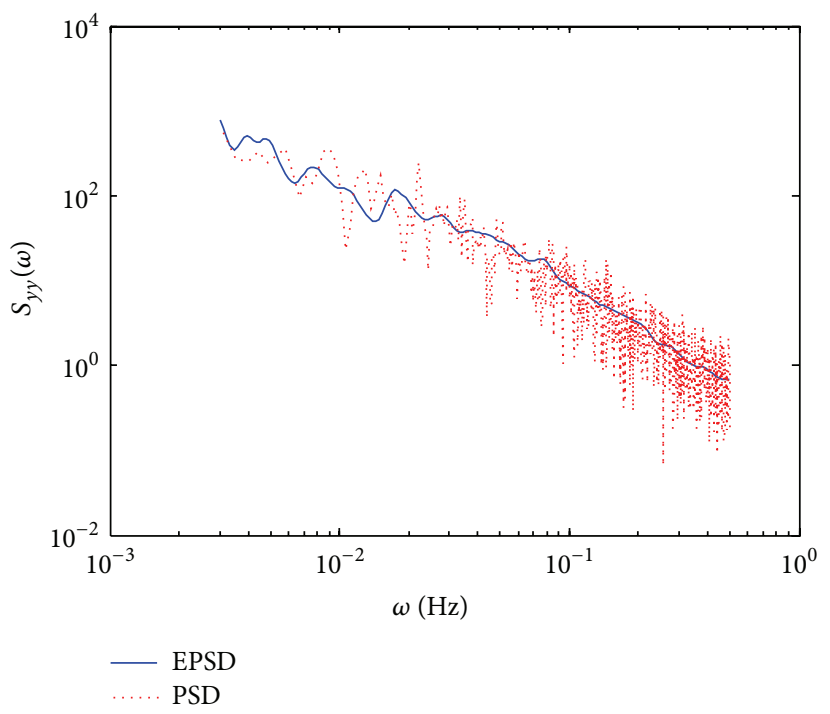

(a) Middle span

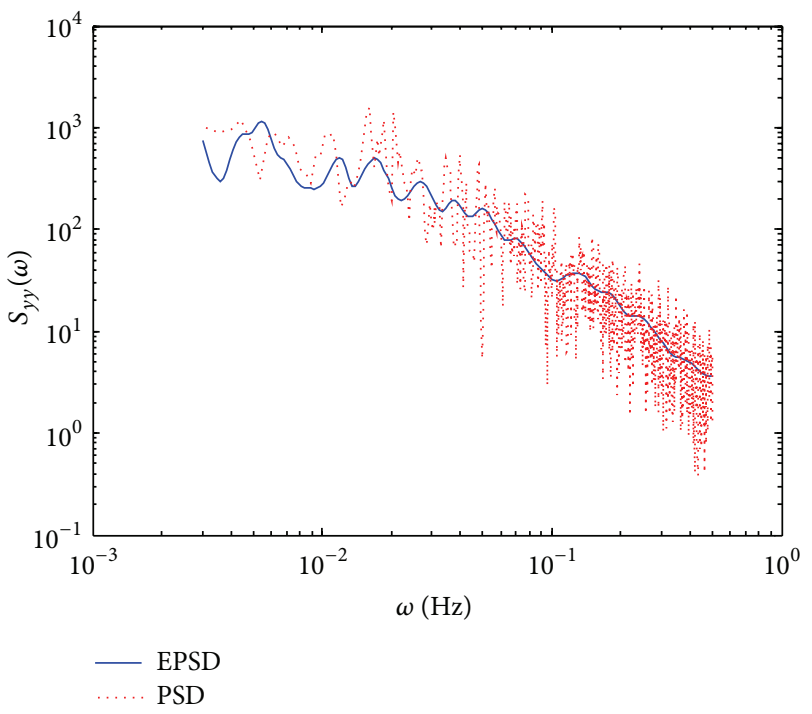

(b) Tower top

FIGURE 6: The marginal feature of estimated EPSD.

4.3. Time-Frequency Characteristics of the Typhoon Matsa. Figure 7 depicts the three-dimensional EPSDs of the Typhoon Matsa in middle span and on tower top. From the figure, the energy distribution of fluctuating wind in both time and frequency domains is clearly illustrated, which cannot be accomplished by traditional Fourier transform. It can be seen that, at different time instants and different frequency points, the energy of fluctuating winds of the Typhoon Matsa has much difference, so the nonstationarity of the Typhoon Matsa is revealed. The wavelet-based EPSD maps energy distribution of the Typhoon Matsa from amplitude-frequency plane to amplitude-frequencytime space, which can help further understanding the time-varying frequency characteristics of nonstationary winds and reasonably predicting structural wind-induced vibrations.

The EPSDs are sliced at different time instants and different frequency points so as to display the detailed energy 


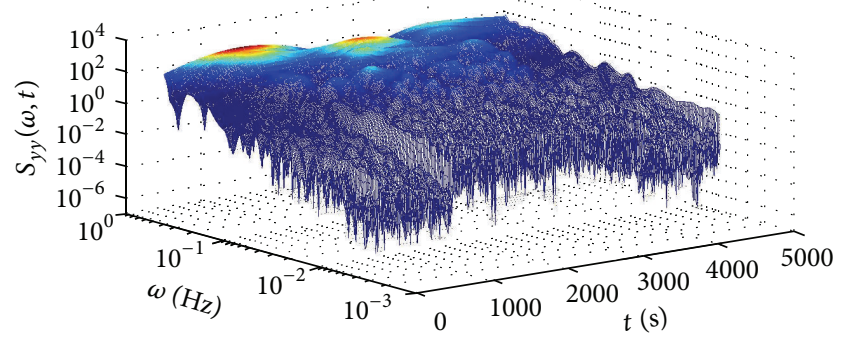

(a) Middle span

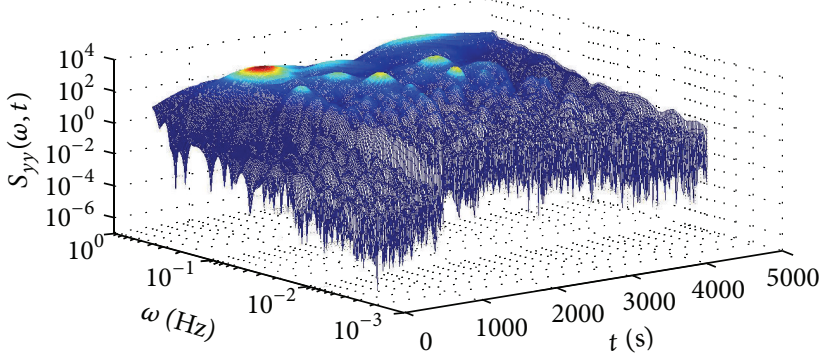

(b) Tower top

Figure 7: 3D view of Typhoon Matsa's EPSD.

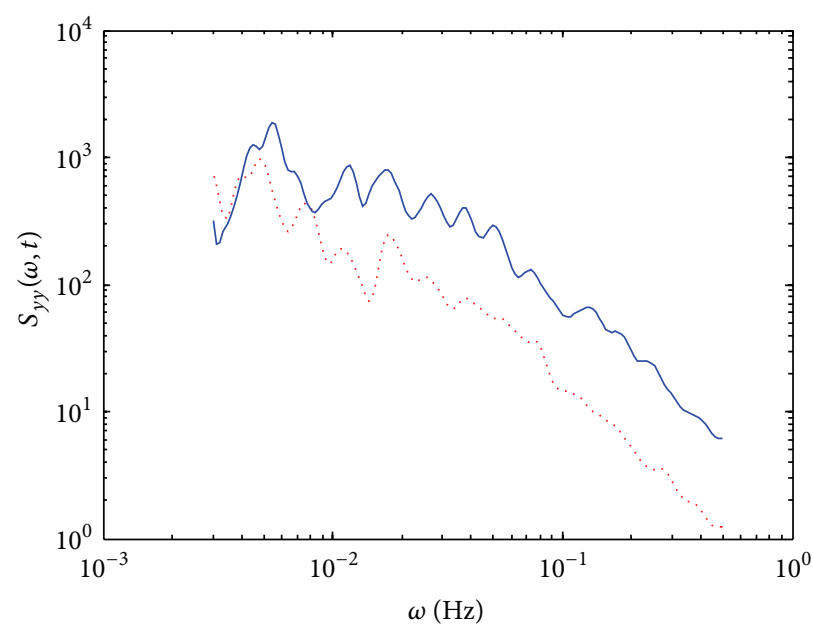

(a) $t=2000 \mathrm{~s}$

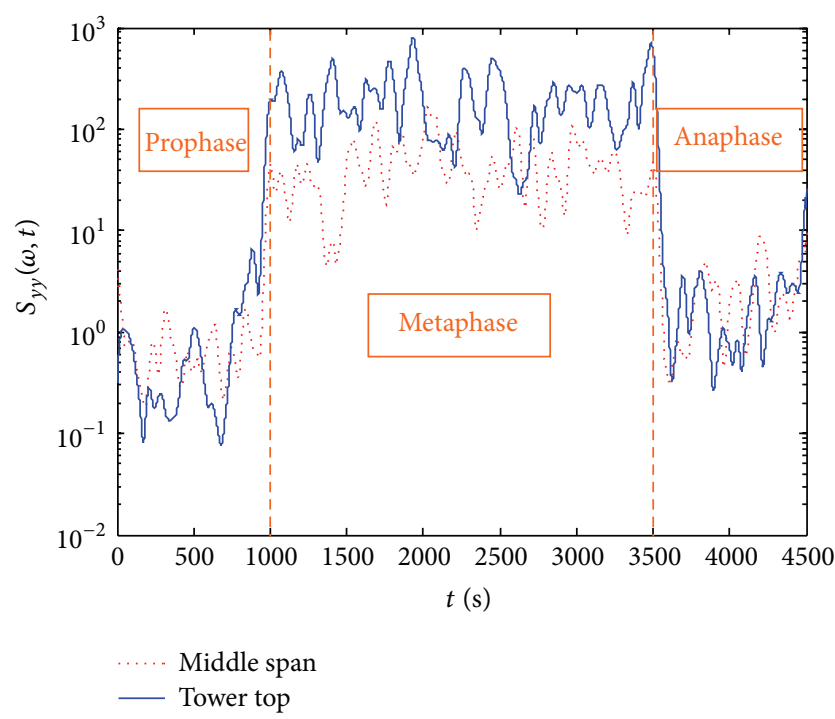

(c) $\omega=0.06 \mathrm{~Hz}$

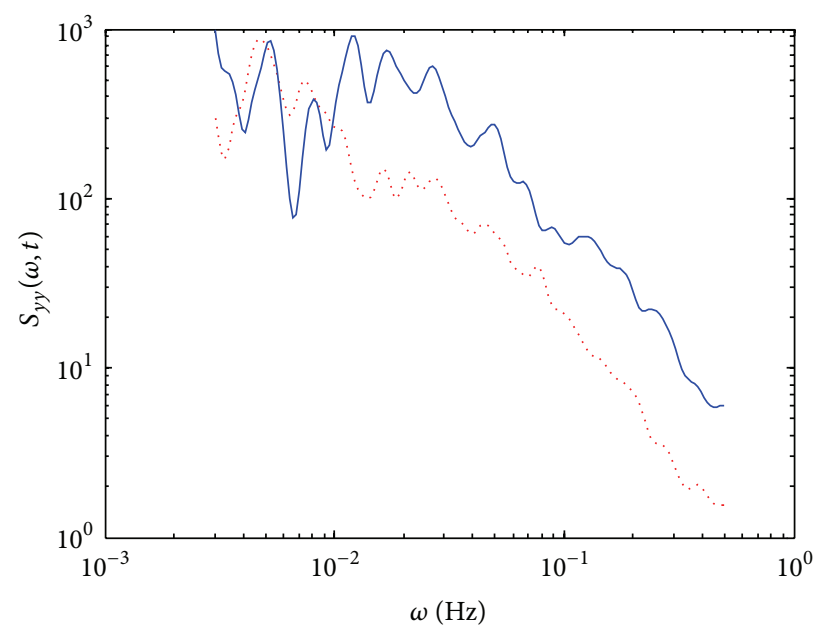

(b) $t=3000 \mathrm{~s}$

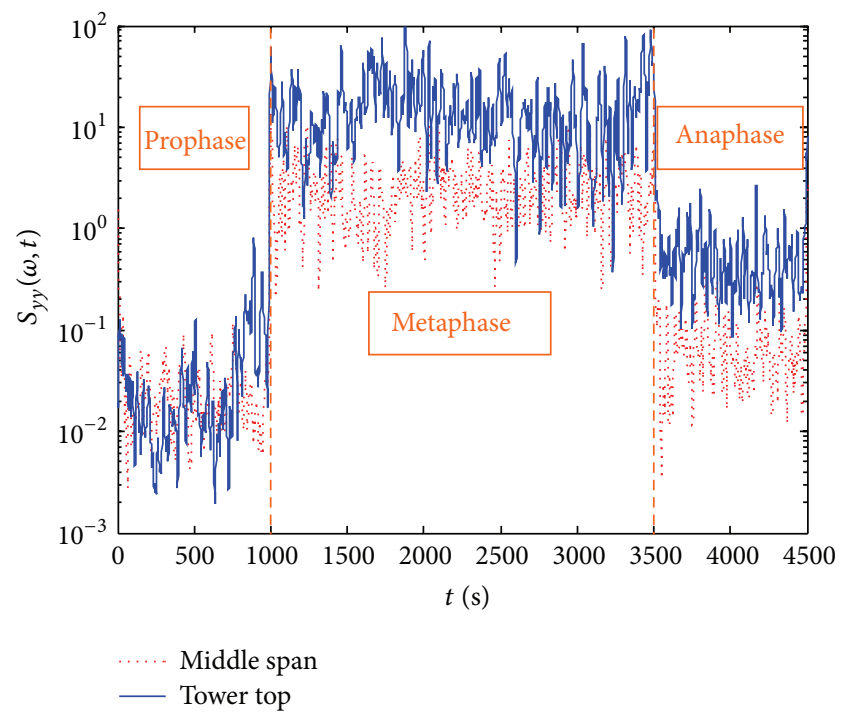

(d) $\omega=0.3 \mathrm{~Hz}$

FIGURE 8: Sliced EPSD of the Typhoon Matsa.

distribution of the Typhoon Matsa. The EPSD slices at time instants of $2000 \mathrm{~s}$ and $3000 \mathrm{~s}$, which belong to the stage of typhoon passing, are shown in Figures 8(a) and 8(b). The results calculated from the data measured in middle span and on tower top are plotted in the same figure for comparing. At the two time instants, the similar feature that the energy of fluctuating wind decreases with the increasing of frequency like the Fourier spectra can be found. When comparing with the results in middle span, the energy of fluctuating wind on tower top is distinctly higher during the typhoon passing. 


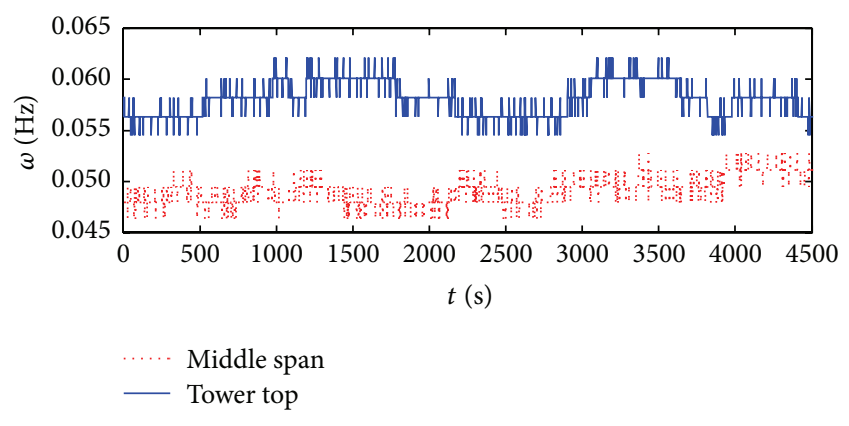

FIgURE 9: Time history of frequency in the Typhoon Matsa.

Figures 8(c) and 8(d) plot the EPSD slices of the Typhoon Matsa at frequencies of $0.06 \mathrm{~Hz}$ and $0.3 \mathrm{~Hz}$. Three stages, namely, prophase, metaphase, and anaphase of the Typhoon Matsa, are enclosed in this figure. The different characteristics in the three stages are revealed. During the prophase, the energy of fluctuating wind in the middle span and on the tower top is low and almost equivalent since the fluctuating wind speed is low and the turbulence energy is relatively small. During the metaphase, the energy of fluctuating wind increases dramatically in the two positions and maintains a high level until the strong wind leaves. The increment of energy on tower top is about 7 times higher than that in middle span, which implies that the higher the structure is, the stronger the typhoon effects are. During the anaphase, the energy of fluctuating wind decreases to the same level as before the strong wind comes. That is to say the influence of the typhoon on the structure is a short-time phenomenon, but the high level of wind force is beyond neglected. The nonstationary characteristics of the fluctuating wind during the typhoon passing are remarkable.

The dominant frequency corresponding to the maximum energy of fluctuating winds at each time instant are plotted in Figure 9. Because the eye of the Typhoon Matsa did not pass the site of the Runyang Suspension Bridge, the variation of frequencies is faint and does not exceed 5\% during the whole process. When investigating other frequency components, a similar conclusion can be deduced. Furthermore, the frequency content has no significant difference in the entire process. So the nonstationary feature of the Typhoon Matsa in frequency domain on this bridge site is weak and can be neglected in practical application.

\section{Conclusions}

The damage or collapse of many structures has close relationship with wind excitations. Investigating time-frequency characteristics of winds especially typhoon processes is a critical issue in structural wind engineering. The EPSD provides an intuitive metric to indicate the energy distribution of typhoon processes at different time instants and different frequency points. Employing the definition of the EPSD and the algorithm of the continuous wavelet transform, a waveletbased EPSD estimation method is developed and applied to the field measured typhoon processes. Some conclusions are drawn as follows.

(1) The EPSD of nonstationary oscillatory processes can be formulated as a sum of time-dependent shape functions modulated by frequency-dependent coefficients. The shape functions are squared moduli of wavelet functions in time domain during the whole duration, and the coefficients relate to the squared values of wavelet coefficients and two wavelet functions with different time shifts.

(2) The EPSD with acceptable accuracy can be estimated by the MO transform, the $\mathrm{MH}$ transform, and the L-P transform. When conducting exact comparison, the $\mathrm{MO}$ transform has stronger ability to capture the nonstationarity of typhoon processes in conjunction with time domain and frequency domain.

(3) The energy of fluctuating winds in the Typhoon Matsa decreases with frequency increasing. During the whole process, the energy of fluctuating typhoon winds is low during the prophase and the anaphase and increases dramatically during the metaphase. The frequency content shows small variation and its shift can be neglected in the Typhoon Matsa.

\section{Conflict of Interests}

The authors declare that there is no conflict of interests regarding the publication of this paper.

\section{Acknowledgments}

The authors wish to gratefully acknowledge Professor T. H. Yi for valuable suggestions and kindly sharing part codes of wavelet transform. This research work was jointly supported by the 973 Program (Grant no. 2015CB060000), the National Natural Science Foundation of China (Grants nos. 51278104 and 51178100), and the Traffic Science Foundation of Jiangsu Province of China (Grant no. 2011Y03).

\section{References}

[1] R. Adhikari and H. Yamaguchi, "A study on the nonstationarity in wind and wind-induced response of tall buildings for adaptive control," in Proceedings of the 9th International Conference on Wind Engineering, vol. 3, pp. 1455-1466, Wiley Eastern Limited, New Delhi, India, 1995.

[2] L. L. Song, J. B. Pang, C. L. Jiang, H. H. Huang, and P. Qin, "Field measurement and analysis of turbulence coherence for Typhoon Nuri at Macao Friendship Bridge," Science China Technological Sciences, vol. 53, no. 10, pp. 2647-2657, 2010.

[3] Y. K. Lin and J. N. Yang, "Multimode bridge response to wind excitations," Journal of Engineering Mechanics-ASCE, vol. 109, no. 2, pp. 586-603, 1983.

[4] D. A. Smith and K. C. Mehta, "Investigation of stationary and nonstationary wind data using classical Box-Jenkins models," Journal of Wind Engineering \& Industrial Aerodynamics, vol. 49, no. 1-3, pp. 319-328, 1993. 
[5] K. Gurley and A. Kareem, "Applications of wavelet transforms in earthquake, wind and ocean engineering," Engineering Structures, vol. 21, no. 2, pp. 149-167, 1999.

[6] T.-H. Yi, H.-N. Li, and M. Gu, "A new method for optimal selection of sensor location on a high-rise building using simplified finite element model," Structural Engineering and Mechanics, vol. 37, no. 6, pp. 671-684, 2011.

[7] T.-H. Yi, H.-N. Li, and M. Gu, "Optimal sensor placement for structural health monitoring based on multiple optimization strategies," The Structural Design of Tall and Special Buildings, vol. 20, no. 7, pp. 881-900, 2011.

[8] J. Chen, M. C. H. Hui, and Y. L. Xu, "A comparative study of stationary and non-stationary wind models using field measurements," Boundary-Layer Meteorology, vol. 122, no. 1, pp. 105-121, 2007.

[9] Y. L. Xu and J. Chen, "Characterizing nonstationary wind speed using empirical mode decomposition," Journal of Structural Engineering, vol. 130, no. 6, pp. 912-920, 2004.

[10] L. Wang and A. Kareem, "Modeling of non-stationary winds in gust-fronts," in Proceeding of the 9th ASCE Joint Specialty Conference on Probabilistic Mechanics and Structural Reliability, CD-ROM, 2004.

[11] Q. S. Li and J. R. Wu, "Time-frequency analysis of typhoon effects on a 79-storey tall building," Journal of Wind Engineering and Industrial Aerodynamics, vol. 95, no. 12, pp. 1648-1666, 2007.

[12] D.-K. Kwon and A. Kareem, "Gust-front factor: new framework for wind load effects on structures," Journal of Structural Engineering, vol. 135, no. 6, pp. 717-732, 2009.

[13] L. Chen and C. W. Letchford, "Numerical simulation of extreme winds from thunderstorm downbursts," Journal of Wind Engineering and Industrial Aerodynamics, vol. 95, no. 9-11, pp. 977990, 2007.

[14] L. Chen, Vector Time-Varying Autoregressive (TVAR) models and their application to downburst wind speeds [Ph.D. thesis], Texas Tech University, Lubbock, Tex, USA, 2006.

[15] L. Wang, Stochastic modelling and simulation of transient events [Ph.D. thesis], University of Notre Dame, 2007.

[16] G. Huang and X. Chen, "Wavelets-based estimation of multivariate evolutionary spectra and its application to nonstationary downburst winds," Engineering Structures, vol. 31, no. 4, pp. 976989, 2009.

[17] L. Hu, Y.-L. Xu, and W.-F. Huang, "Typhoon-induced nonstationary buffeting response of long-span bridges in complex terrain," Engineering Structures, vol. 57, pp. 406-415, 2013.

[18] E. P. Wigner, "On the quantum correction for thermodynamic equilibrium," Physical Review, vol. 40, no. 5, pp. 749-759, 1932.

[19] J. Ville, "Theorie et applications de la notion de signal analytique," Cables and Transmission, vol. 2, pp. 61-74, 1948.

[20] G. Failla, M. Pappatico, and G. A. Cundari, "A wavelet-based spectrum for non-stationary processes," Mechanics Research Communications, vol. 38, no. 5, pp. 361-367, 2011.

[21] R. G. Stockwell, L. Mansinha, and R. P. Lowe, "Localization of the complex spectrum: the S transform," IEEE Transactions on Signal Processing, vol. 44, no. 4, pp. 998-1001, 1996.

[22] R. G. Stockwell, S-transform analysis of gravity wave activity from a small scale network of airglow imagers [Ph.D. thesis], University of Western Ontario, Ontario, Canada, 1999.

[23] S. G. Mallat, "A theory for multiresolution signal decomposition: the wavelet representation," IEEE Transactions on Pattern Analysis and Machine Intelligence, vol. 11, no. 7, pp. 674-693, 1989.
[24] T.-H. Yi, H.-N. Li, and X.-Y. Zhao, "Noise smoothing for structural vibration test signals using an improved wavelet thresholding technique," Sensors (Switzerland), vol. 12, no. 8, pp. 11205-11220, 2012.

[25] K. V. Nguyen, "Comparison studies of open and breathing crack detections of a beam-like bridge subjected to a moving vehicle," Engineering Structures, vol. 51, pp. 306-314, 2013.

[26] T.-H. Yi, H.-N. Li, and M. Gu, "Wavelet based multi-step filtering method for bridge health monitoring using GPS and accelerometer," Smart Structures and Systems, vol. 11, no. 4, pp. 331-348, 2013.

[27] Z. H. Min and L. M. Sun, "Wavelet-based structural modal parameter identification," Structural Control and Health Monitoring, vol. 20, no. 2, pp. 1-18, 2013.

[28] P. D. Spanos and G. Failla, "Evolutionary spectra estimation using wavelets," Journal of Engineering Mechanics, vol. 130, no. 8, pp. 952-960, 2004.

[29] A. Moghtaderi, G. Takahara, and D. J. Thomson, "Evolutionary spectrum estimation for uniformly modulated processes with improved boundary performance," in Proceedings of the IEEE International Conference on Acoustics, Speech, and Signal Processing (ICASSP '09), pp. 2993-2996, April 2009.

[30] M. B. Priestley, "Power spectral analysis of non-stationary random processes," Journal of Sound and Vibration, vol. 6, no. 1, pp. 86-97, 1967.

[31] M. Shinozuka and C.-M. Jan, "Digital simulation of random processes and its applications," Journal of Sound and Vibration, vol. 25, no. 1, pp. 111-128, 1972.

[32] M. Shinozuka, C.-B. Yun, and H. Seya, "Stochastic methods in wind engineering," Journal of Wind Engineering and Industrial Aerodynamics, vol. 36, no. 1-3, pp. 829-843, 1990.

[33] B. Chen, J. Zheng, and W. L. Qu, "Control of wind-induced response of transmission tower-line system by using magnetorheological dampers," International Journal of Structural Stability and Dynamics, vol. 9, no. 4, pp. 661-685, 2009.

[34] B. Chen, W. H. Guo, P. Y. Li, and W. P. Xie, "Dynamic responses and vibration control of the transmission tower-line system: a state-of-the-art-review," The Scientific World Journal, vol. 2014, Article ID 538457, 20 pages, 2014.

[35] T.-H. Yi, H.-N. Li, and X.-D. Zhang, "Sensor placement on Canton Tower for health monitoring using asynchronous-climb monkey algorithm," Smart Materials and Structures, vol. 21, no. 12, Article ID 125023, 2012. 


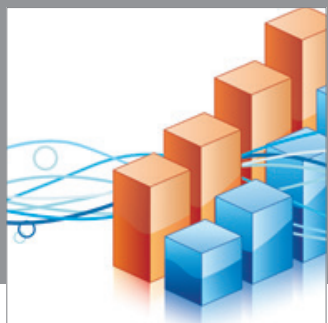

Advances in

Operations Research

mansans

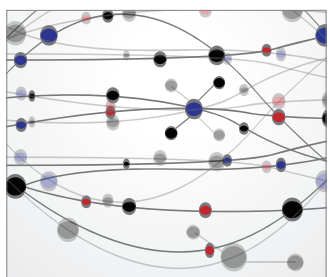

The Scientific World Journal
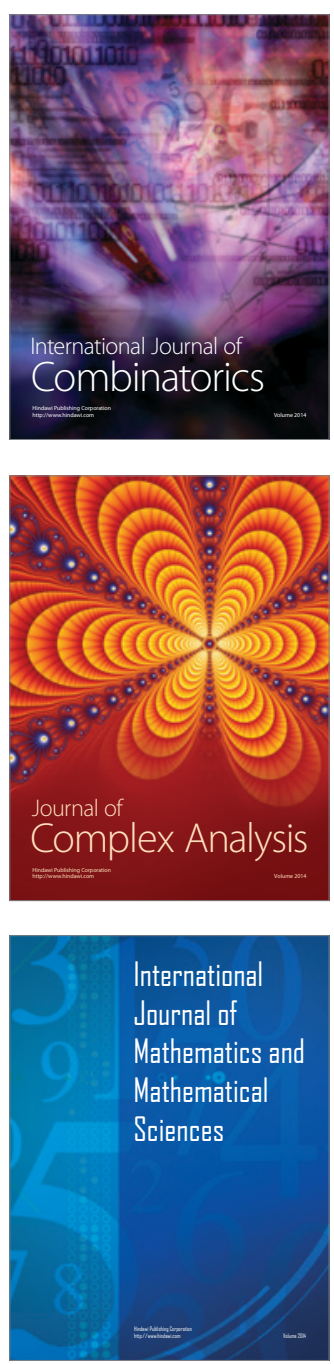
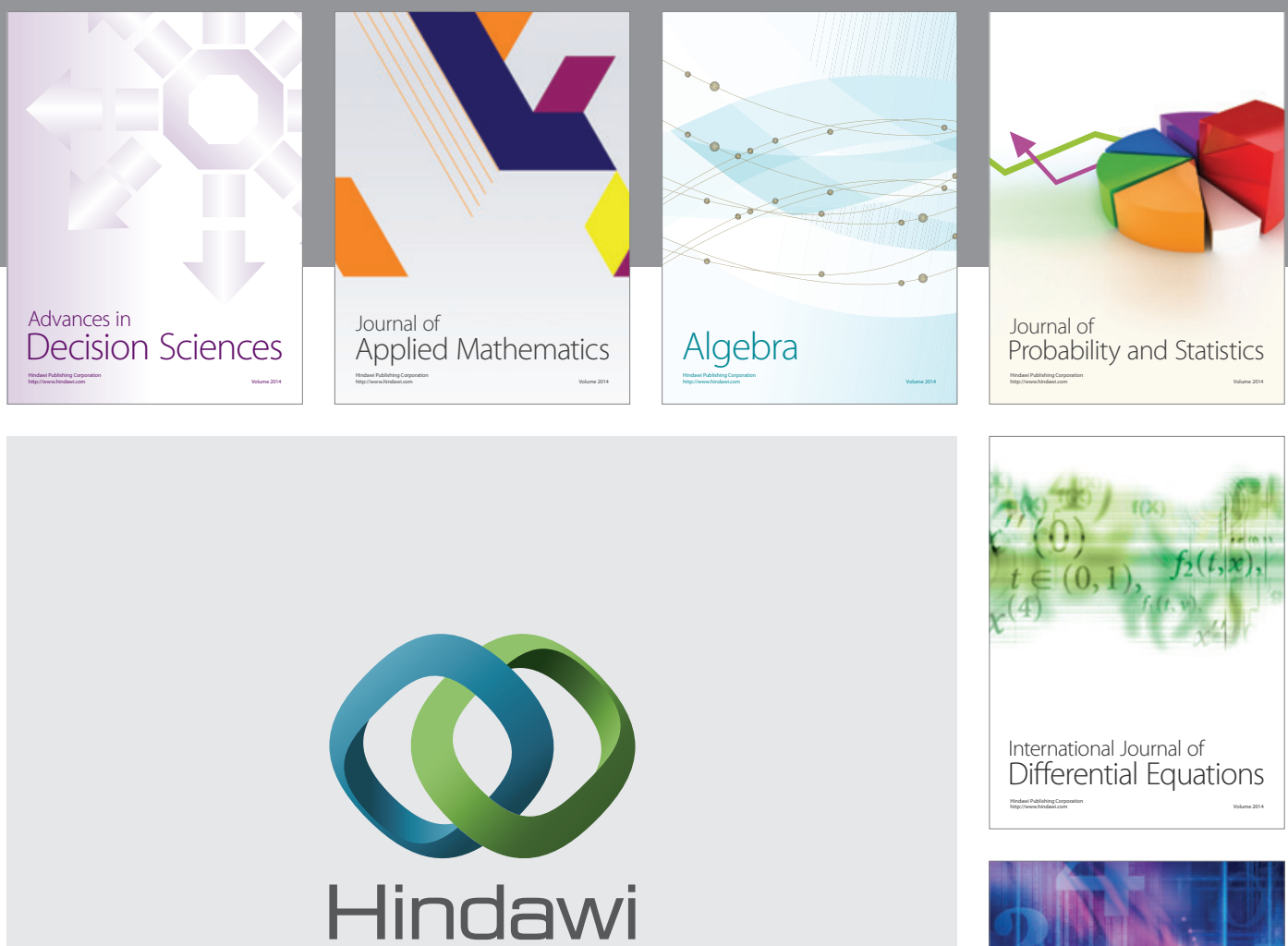

Submit your manuscripts at http://www.hindawi.com
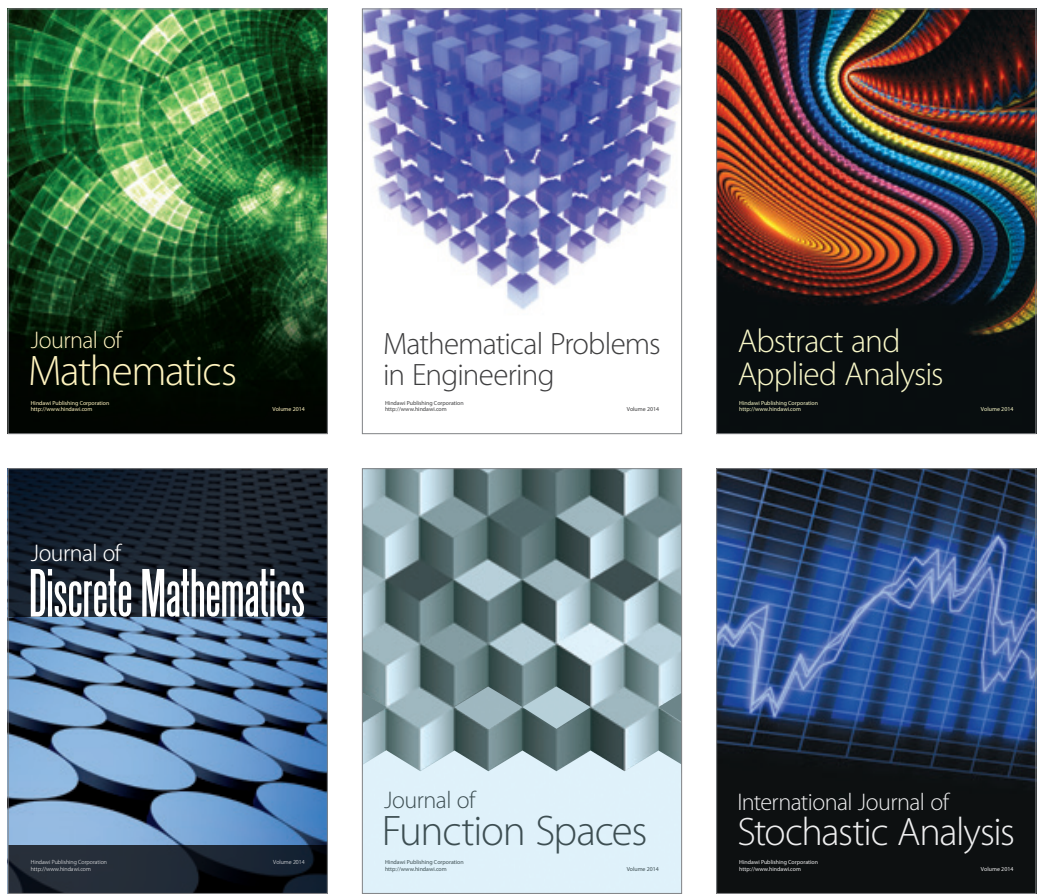

Journal of

Function Spaces

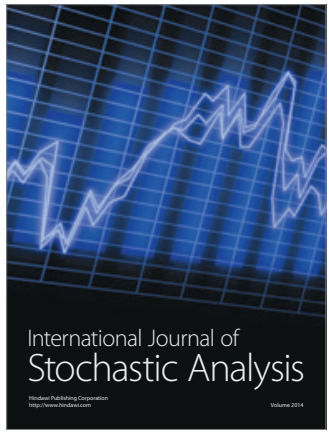

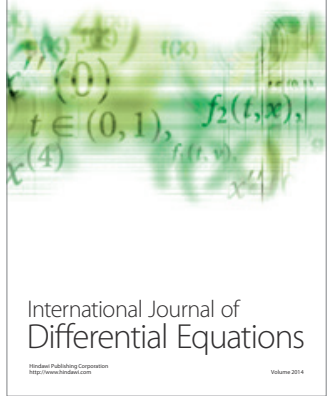
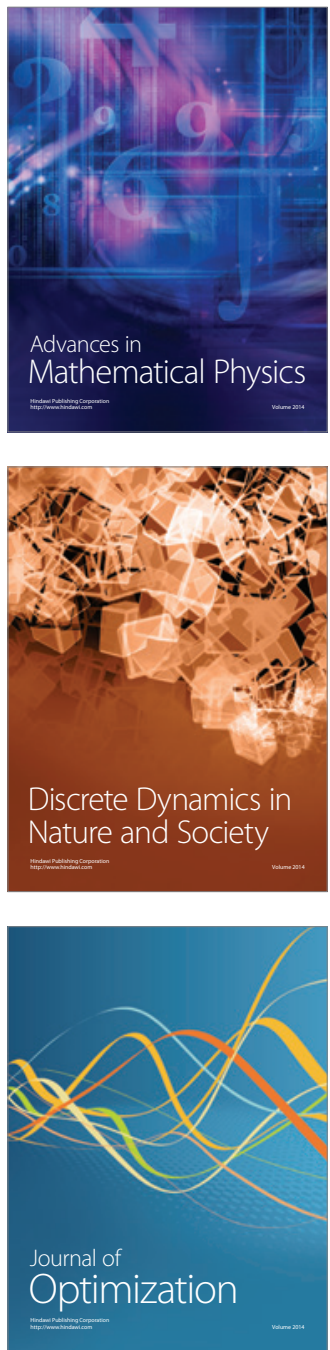